\title{
Morfometría y Variantes Anatómicas del Tronco Celíaco en Población Mexicana
}

\author{
Morphometry and Anatomical Variants of the Celiac Trunk in Mexican Population
}

Pineda Martínez, D. '; Vargas Figueroa, A. I. 1; Sosa Castillo, H. S. ' ; Martínez Anda, J. J. ; Avendaño Pradel, R. ${ }^{3}$; Estrada León, R. A. ${ }^{1}$; Alanís Mendizábal, J. ${ }^{1}$ \& Valencia-Caballero, L. ${ }^{1}$

PINEDA, M. D.; VARGAS, F. A. I.; SOSA, C. H. S.; MARTÍNEZ, A. J. J.; AVENDAÑO, P. R.; ESTRADA, L. R. A.; ALANÍS, M. J. \& VALENCIA-CABALLERO, L. Morfometría y variantes anatómicas del tronco celíaco en población mexicana. Int. J. Morphol., 37(1):174$177,2019$.

RESUMEN: Las variantes anatómicas del tronco celíaco (TC) son relevantes en el ámbito quirúrgico e intervencionista. Existen diferentes estudios a nivel mundial que han demostrado las variaciones que puede presentar el TC tanto en longitud como en estructura; dichos estudios han sido realizados predominantemente en población asiática y europea. Por lo anterior, realizamos un estudio que caracterizó esta estructura y que sea referente para la población mexicana. Se analizó una muestra de 50 especímenes cadavéricos humanos embalsamados de origen mexicano. El promedio de longitud del TC a su primera rama fue de $12,44 \mathrm{~mm}$; de su origen a la segunda rama fue de $17,07 \mathrm{~mm}$; y hasta la tercera fue de 19,15 mm. En la muestra de estudio se encontraron variantes en el $20 \%$ de los especímenes, de éstos el $14 \%$ presentaron variantes morfométricas en cuanto a longitud y $6 \%$ en estructura. Respecto a las variantes morfométricas, destacó la presencia de un TC de $3 \mathrm{~mm}$ de longitud. En cuanto a las variantes morfológicas dos individuos presentaron un tronco gastro-esplénico con tronco hepato-mesentérico; y el tercero un tronco bifurcado hepato-gástrico con la arteria esplénica naciendo de la arteria hepática común. Éste último no reportado en la literatura.

PALABRAS CLAVE: Variantes anatómicas; Tronco celíaco; Población mexicana; Morfometría; Morfología.

\section{INTRODUCCIÓN}

El conocimiento de las variantes anatómicas del tronco celíaco (TC) es de vital importancia en el ámbito quirúrgico e intervencionista pues disminuye el riesgo de mortalidad asociado a procedimientos quirúrgicos iatrogénicos derivados de la falta de conocimiento de dicha estructura. Existen diferentes estudios a nivel mundial en los que predomina el análisis de muestras en población asiática y europea, estos han mostrado las variantes que puede presentar el TC; pero ninguno ha sido realizado en población mexicana. Por ello, decidimos realizar un estudio que describa la morfometría, morfología y refleje las variantes anatómicas existentes en población mexicana.

El estudio de las variantes del TC requiere conocimiento de su desarrollo embriológico; a medida que el intestino y mesenterio comienzan a estrecharse y la aorta dorsal comienza a fusionarse, la 10a, 11a y 12a ramas segmentarias ventrales también lo hacen hacia la línea me- dia; al ocurrir este proceso se forma el tronco celíaco y sus ramas principales (Alakkam et al., 2016).

En 1756, Haller describe por primera vez la morfología del TC (Al Awad et al., 2012). Él menciona que éste emerge de la aorta abdominal (AA) después del hiato aórtico del diafragma, a nivel de la $12^{\mathrm{a}}$ vértebra torácica y $1^{\mathrm{a}}$ vértebra lumbar dando un corto recorrido anteroinferior y originando tres ramas: la arteria gástrica izquierda (AGI) que se dirige a la unión cardio-esofágica para dar origen a algunas ramas esofágicas, y dirigirse hacia abajo sobre la curvatura menor del estómago hasta anastomosarse con la arteria gástrica derecha. La arteria esplénica (AE) se dirige hacia la izquierda por el margen superior del páncreas hasta el ligamento espleno-renal dando origen a las ramas gástricas cortas y gastro-omental izquierda, para después dividirse e ingresar al bazo. La arteria hepática común (AHC) se dirige a la derecha para dar origen a la rama gástrica derecha y des-

\footnotetext{
${ }^{1}$ Departamento de Anfiteatro, Facultad de Medicina, Universidad Nacional Autónoma de México, México.

${ }^{2}$ Centro Médico ABC. México.

${ }^{3}$ Facultad de Ciencias de la Salud Universidad Anáhuac México Norte, México.
} 
pués se bifurca en dos ramas terminales: la arteria gastroduodenal que brinda vascularización al páncreas y al duodeno proximal; y la arteria hepática propia la cual irriga al hígado y la vía biliar (Drake 2015; Moore et al., 2015).

Conociendo el origen embriológico y la anatomía de estas arterias sabemos que al momento de fusionarse pueden hacerlo de distintas formas, surgiendo así las variantes anatómicas de importancia clínico-quirúrgicas.

\section{MATERIAL Y MÉTODO}

En el Departamento de Anfiteatro de la Facultad de Medicina de la Universidad Nacional Autónoma de México (UNAM) se analizó una muestra de 50 cadáveres humanos (34 hombres y 16 mujeres) de origen mexicano, de ambos sexos y con un rango de edad entre 30 y 90 años (edad promedio al fallecimiento 67 años). Todos los cadáveres estaban preparados con técnicas de conservación. En cada espécimen se realizó una incisión tipo Chevron (Chute et al., 1968), por planos para abordar la cavidad abdominal; se identificó la curvatura menor del estómago, el ligamento hepatogástrico y el ligamento hepato-duodenal con apertura del mismo. Se disecó la tríada portal para identificar la arteria hepática propia y seguir su trayecto hacia la línea mediana para encontrar la AHC y posteriormente el TC. Una vez localizado éste último se siguieron sus ramas principales (AGI y AE) hasta los órganos de su desembocadura.

Identificado el tronco celíaco se realizó la toma de medidas utilizando un vernier, tomando como puntos de referencia el origen del TC en la parte abdominal de la aorta hasta el punto medio del nacimiento de cada rama. Cabe mencionar que la longitud del TC fue considerada desde su origen a la primera rama, independientemente cual fuera.

Tabla I. Análisis morfométrico del tronco celíaco de acuerdo con la longitud de sus ramas.

\begin{tabular}{lccccc}
\hline & & Mín & Máx & Medi & DT \\
\hline Arteria & $\mathrm{n}$ & $\mathrm{mm}$ & $\mathrm{mm}$ & $\mathrm{mm}$ & $\mathrm{mm}$ \\
\hline Tronco celíaco & 47 & 3 & 30 & 12,44 & 4,92 \\
Segunda Rama & 47 & 3 & 36 & 17,07 & 6,36 \\
Tercera Rama & 47 & 3 & 40 & 19,15 & 6,93 \\
\hline
\end{tabular}

Tabla II. Frecuencia de orden de aparición de las ramas del tronco celíaco que no presentaron variantes estructurales.

\begin{tabular}{lccc}
\hline Emergencia de ramas del TC. & AGI & AE & AHC \\
\hline $1^{\text {era }}$ Rama & $98 \%$ & $2 \%$ & $0 \%$ \\
$2^{\text {da }}$ Rama & $2 \%$ & $96 \%$ & $2 \%$ \\
$3^{\text {ra }}$ Rama & $0 \%$ & $2 \%$ & $98 \%$ \\
\hline
\end{tabular}

Se documentaron las disecciones a través de fotografía digital con el equipo Nikon D3300. Posteriormente se conformó una base de datos para realizar el registro y análisis estadístico descriptivo con el programa SPSS 21 (Propiedad IBM Corp.).

\section{RESULTADOS}

Del total de la muestra se analizó la morfometría del TC en 47 individuos, y en los tres sujetosrestantes se estudió de manera independiente el TC debido a que presentaron variaciones anatómicas.

En los 47 individuos, el promedio de longitud del TC desde su origen hasta la primera rama fue de $12,44 \mathrm{~mm}$, del origen del tronco hasta su segunda rama fue de 17,07 $\mathrm{mm}$ y, por último, del origen del TC hasta su tercera rama fue de 19,15 mm (Tabla I).

Respecto a la frecuencia del nacimiento de las ramas la AGI fue la primera rama del TC en el $98 \%$, la AE fue la segunda en el $96 \%$ y la AHC fue la tercera en el $98 \%$ del total de la muestra (Tabla II).

En 50 muestras se encontró un $20 \%$ de variantes tanto longitudinales como de estructura. Es decir, siete individuos presentaron variantes morfométricas $(14 \%)$ y tres en cuanto a su morfología (6\%).

De los siete especímenes que presentaron variantes anatómicas relevantes respecto a su longitud (promedio \pm 2 D.E. de nuestra muestra); en uno se encontró variabilidad significativa respecto a la distancia desde su origen a su primera división, y en el resto se encontró variabilidad significativa respecto a la distancia desde su origen a la segunda y tercera ramas. Destaca la presencia de un TCA de un tercio de longitud (Fig. 1).

En los tres especímenes con variantes estructurales, dos $(4 \%)$ presentaron un tronco gastro-esplénico con tronco hepato-mesentérico y en uno (2\%) se identificó un tronco bifurcado hepato-gástrico con la arteria esplénica naciendo de la arteria hepática común.

A pesar de no incluir a estos tres sujetos en el análisis morfométrico global, se registró la longitud de sus estructuras. En los especímenes que presentaron tronco hepato-mesentérico con tronco gastro-esplénico, los promedios de longitud fueron desde su origen, hasta su primera rama (AGI): $24 \mathrm{~mm}$, hasta su segunda rama (AE): 26,5 $\mathrm{mm}$; entre el origen del tronco hepato-mesentérico y el 


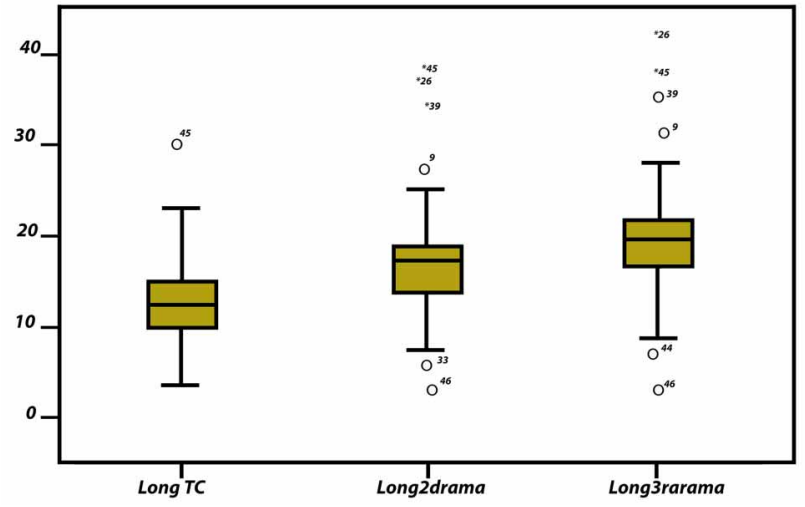

Fig. 1. Se presenta como caja las medias de longitud dentro de cada rama del TC. Aquellas que presentaron una desviación estándar +2 fueron consideradas variantes de longitud. Cada punto tiene un superíndice señalando el número de espécimen al cual corresponde.
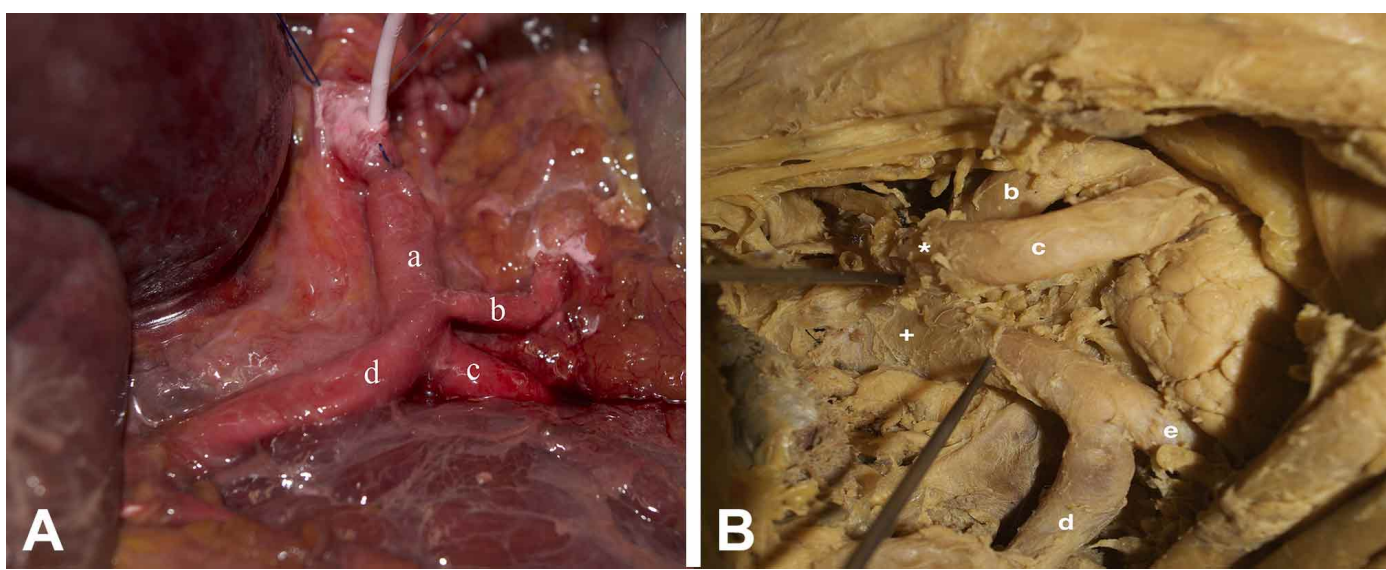

Fig. 2. B). Variante estructural: tronco gastro-esplénico con tronco hepato-mesentérico (b) arteria gástrica izquierda (c) arteria esplénica, (d) arteria hepática común, (e) arteria mesentérica superior, $(*)$ tronco gastroesplénico, (+) tronco hepato-mesentérico.

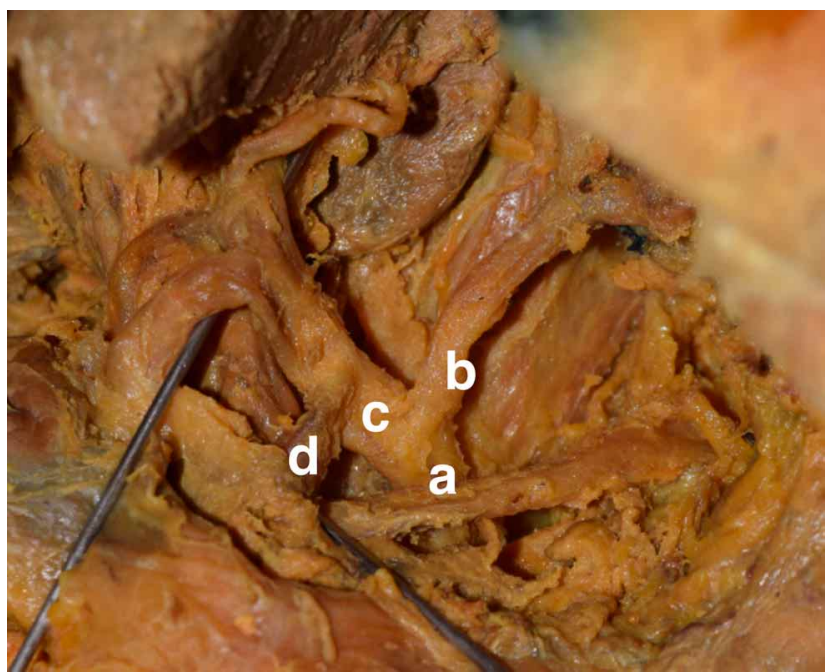

Fig. 3. Tronco hepato-gástrico con arteria esplénica naciendo de la arteria hepática común (a) tronco hepato-gástrico; (b) arteria gástrica izquierda; (c) arteria hepática común; (d) arteria esplénica. tronco gastro-esplénico: $6 \mathrm{~mm}$. Del origen del tronco hepatomesentérico a su primera rama la AHC: $27 \mathrm{~mm}$, a su segunda rama, arteria mesentérica superior: 28,5 mm (Fig. 2).

En el espécimen con tronco hepato-gástrico con AE originándose de $\mathrm{AHC}$, la longitud del tronco hepato-gástrico a su bifurcación en AHC y AGI fue de $7 \mathrm{~mm}$. La AE nació desde la $\mathrm{AHC}$ a $8 \mathrm{~mm}$ de su bifurcación en el tronco, sumando un total de $15 \mathrm{~mm}$ desde el origen del tronco hepato-gástrico hasta el nacimiento de la AE desde la AHC (Fig. 3).

\section{DISCUSIÓN}

En la literatura existen varios repórteres del TC realizados a través de estudios de imagen o transoperatorios (Panagouli et al., 2013; Song et al., 2010; López-Andújar et al., 2007). La bibliografía reporta la presencia de un TC trifurcado en el 89,42 \% (Panagouli et al.) levemente superior en nuestro estudio (94\%). Por otro lado, Song et al., reportaron la presencia de variantes anatómicas estructurales en el 9,64\%, mientras que en nuestra muestra fue de $6 \%$.

En algunos estudios la raza y sexo no suelen considerarse, sin embargo, en los que se reportan, predomina el sexo masculino, así como los grupos raciales caucásico y mongoloide (japonés) (Panagouli et al.).

Existen diferentes clasificaciones estructurales del TC y sus ramas principales, entre las más utilizadas por su aplica- 
ción quirúrgica se encuentran la de Michel y las de Adachi (Panagouli et al.). Estas clasificaciones son de relevancia para especialistas en cirugía hepática, pancreática, gástrica, biliar, cirugía de trasplante y para cirujanos generales involucrados en procedimientos abiertos y laparoscópicos de la porción superior del abdomen, así como radiólogos intervencionistas.

Una de las variantes estructurales encontrada en dos especímenes corresponde al Tipo V: tronco gastro-esplénico con tronco hepato-mesentérico de la clasificación de Adachi (Panagouli et al.) y otra no está reportada en la literatura, consistió en un tronco bifurcado, donde la AGI y AHC se originaban de un mismo tronco, mientras que la $\mathrm{AE}$ se originaba de la AHC. Proponemos considerarla como una variante estructural. La denominación sugerida es: "Tronco hepato-gástrico con arteria esplénica originándose de la arteria hepática común".

En los estudios citados, la longitud reportada del TC hasta su primera rama va desde $10 \mathrm{~mm}$ hasta $41 \mathrm{~mm}$ (Severino, et al., 2014). En nuestro trabajo se encontró una longitud de 3 a $30 \mathrm{~mm}$, lo cual contrasta con los reportes previos.

Con este estudio brindamos una caracterización morfológica del TC, estableciendo un estándar de medición para cada una sus ramas, así como el reporte de las variantes morfométricas y morfológicas presentes en una muestra de población mexicana. Este estudio contrasta con la literatura internacional, por lo que pretende establecer un referente estructural y morfométrico del TC y sus ramas en población mexicana con la finalidad de disminuir los procedimientos iatrogénicos relacionados con esta estructura.

AGRADECIMIENTOS. A los auxiliares forenses del Departamento de Anfiteatro, de la Facultad de Medicina de la UNAM: Rubén Eduardo Durazno López, Gonzalo Mejía Medina y Luis Fernando García González.

PINEDA, M. D.; VARGAS, F. A. I.; SOSA, C. H. S.; MARTÍNEZ, A. J. J.; AVENDAÑO, P. R.; ESTRADA, L. R. A.; ALANíS, M. J. \& VALENCIA-CABALLERO, L. Morphometry and anatomical variants of the celiac trunk in a Mexican population. Int. J. Morphol., 37(1):174-177, 2019.

SUMMARY: Anatomical variants in the celiac trunk (CT) are important in surgical and interventional fields. Studies worldwide have demonstrated length and structure variations in the celiac trunk. These studies have predominantly been carried out in Asian and European population. Therefore, we considered it necessary to realize a study to describe this structure and serve as a reference in the Mexican population. A sample of 50 human cadaveric specimens of Mexican origin was analyzed. The average length of the celiac trunk from its origin to its first branch was $12.44 \mathrm{~mm}$, to the second branch was $17.07 \mathrm{~mm}$, and to the third branch was $19.15 \mathrm{~mm}$. In the studied sample, variants were found in $20 \%$ of the specimens, $14 \%$ of these presented morphometric variations in terms of length, and $6 \%$ in terms of structure. In reference to the morphometric variants, the presence of one $3 \mathrm{~mm}$ length CT was noteworthy. Morphological variants were found in two specimens presenting a gastro-splenic trunk with a hepatomesenteric trunk, and a third specimen with hepatogastric bifurcated trunk, with the splenic artery originating from the common hepatic artery was observed. No reports were found in the literature concerning the latter.

KEY WORDS: Anatomical variations; Celiac trunk; Mexican population; Morphometry; Morphology.

\section{REFERENCIAS BIBLIOGRÁFICAS}

Al Awad, A.; Villasmil, O.; Muñoz Cabas, D.; Villasmil, P.;Ayubi, A.; Castellano, S.; Chacón, C.; Paz , J. \& Chacón, J. Variante anatómica de la arteria hepática a nivel de la placa hiliar: A propósito de un caso. Rev. Argent. Anat. Online, 3(1):14-7, 2012.

Alakkam, A.; Hill, R. V. \& Saggio, G. Superior mesenteric origin of the proper hepatic artery: embryological and clinical implications. Surg. Radiol. Anat., 38(6):747-50, 2016.

Chute, R.; Baron, J. A. Jr. \& Olsson, C. A. The transverse upper abdominal "chevron" incision in urological surgery. J. Urol., 99(5):528-32, 1968.

Drake, R. Gray. Anatomía para Estudiantes. $3^{\text {rd }}$ ed. St. Louis, Elsevier Mosby, 2015.

López-Andújar, R.; Moya, A.; Montalvá, E.; Berenguer, M.; De Juan, M.; San Juan, F.; Pareja, E.; Vila, J. J.; Orbis, F.; Prieto, M. \& Mir, J. Lessons learned from anatomic variants of the hepatic artery in 1,081 transplanted livers. Liver Transpl., 13(10):1401-4, 2007.

Moore, K. L.; Dalley, A. F. \& Agur, A. M. R. Clinically Oriented Anatomy. $7^{\text {th }}$ ed. Philadelphia, Wolters Kluwer Health/Lippincott Williams \& Wilkins, 2015.

Panagouli, E.; Venieratos, D.; Lolis, E. \& Skandalakis, P. Variations in the anatomy of the celiac trunk: A systematic review and clinical implications. Ann. Anat., 195(6):501-11, 2013.

Song, S. Y.; Chung, J. W.; Yin, Y. H.; Jae, H. J.; Kim, H. C.; Jeon, U. B.; Cho, B. H.; So, Y. H. \& Park, J. H. Celiac axis and common hepatic artery variations in 5002 patients: systematic analysis with spiral CT and DSA. Radiology, 255(1):278-88, 2010.

Dirección para correspondencia:

Dra. Lorena Valencia Caballero

Departamento de Anfiteatro

Basamento del Edificio B

Facultad de Medicina

Universidad Nacional Autónoma de México.

Av. Universidad 3000

Ciudad Universitaria. C. P.: 04510

Delegación: Coyoacán

Ciudad de México

MÉXICO

Email: loreval@unam.mx 